
gestion compartida con el emprendedor social en empresas mixtas del sector petrolero. Económicas CUC, 37(2), 4768. DOI: http://dx.doi.org/10.17981/econcuc.37.2.2016.03

\title{
Responsabilidad social gestión compartida con el emprendedor social en empresas mixtas del sector petrolero ${ }^{1}$
}

DOI: http://dx.doi.org/10.17981/econcuc.37.2.2016.03

\author{
Annherys Paz Marcano ${ }^{2}$ \\ Jaiham Harris ${ }^{3}$ \\ Félix Franco Segovia ${ }^{4}$
}

\section{Resumen}

El objetivo del artículo es analizar la responsabilidad social como gestión compartida con el emprendedor social en empresas mixtas del sector petrolero de Venezuela. Metodológicamente, se establece un tipo de estudio analítico, descriptivo y de campo. Diseño de investigación no experimental, transaccional. Para la población se seleccionaron (30) treinta gerentes de las empresas objeto de estudio. La técnica de recolección de datos fue la encuesta, y como instrumento dos cuestionarios con escala de estimación tipo múltiples, con una ponderación del 1 al 5 y un índice de confiabilidad Alpha Cronbach ubicado en 0,94 para el cuestionario responsabilidad social y 0.93 para la variable emprendedor social. Los resultados indicaron que la responsabilidad social, representa una gestión compartida con el emprendedor social en empresas objeto de estudio, siendo constructores de comunidades sostenibles a favor de mejorar el bienestar del entorno. Se resalta como conclusión, que el emprendedor social debe ser una persona integral desde un enfoque creativo, innovador, tolerante, responsable, visionario, capaz de forjar ideas que ayuden a minimizar aquellos efectos que incidan sobre su gestión voluntaria.

\footnotetext{
${ }^{1}$ Artículo de tipo científico; Investigación Base Responsabilidad Social y Emprendedor Social en Empresas Mixtas del Sector Petrolero. Línea de Investigación: Ética y responsabilidad social en las Organizaciones .Maestría Gerencia Empresarial. Adscrito a la Direccion de investigación y Postgrado Universidad Rafael Belloso Chacín (URBE). Maracaibo Venezuela

${ }^{2}$ Posdoctora en Gerencia de las Organizaciones. Doctora en Ciencias Gerenciales. Magister en Gerencia de Recursos Humanos. Lcda. En Administración de Empresas. Universidad Rafael Belloso Chacin - Venezuela. Profesora e Investigadora adscrita al grupo AIKA de la Universidad de la Guajira. Autor de correspondencia, aipaz@uniguajira.edu.co

${ }^{3}$ Posdoctora en Gerencia Pública y Gobierno, Doctora en Ciencias Gerenciales, Magister en Gerencia de Recursos Humanos, Lcda. En Administración de Empresas. Universidad Rafael Belloso Chacin - Venezuela. Profesor titular e investigadora de la Universidad Rafael Belloso Chacín - Maracaibo, Venezuela. (Pdte Grupo de Investigación). jaihamharris@hotmail.com

${ }^{4}$ Doctor en Ciencias Gerenciales. Magister en Gerencia Empresarial. Universidad Rafael Belloso Chacín - Venezuela. Lcdo. en Administración mención Gerencia Industrial. Universidad Nacional Experimental Rafael María Baralt, UNERMB Venezuela. Profesor universitario e investigador (Pdte Universidad - Grupo de Investigación). fjfs67@hotmail.com
} 
Palabras clave: responsabilidad social; gestión compartida; emprendedor social; empresas mixtas del sector petrolero; Venezuela.

\title{
Social responsibility shared management with the social entrepreneur in mixed companies in oil sector
}

\begin{abstract}
The objective of the article is to analyze social responsibility as shared management with the social entrepreneur in mixed companies of the oil sector of Venezuela. Methodologically, a type of analytical, descriptive and field study is established, design of non-experimental, transactional research. For the population, thirty (30) managers of the companies under study were selected. The data collection technique was a survey, and as an instrument two questionnaires with multiple scale estimation, with a weighting of 1 to 5 and an Alpha Cronbach reliability index located at 0.94 for the social responsibility questionnaire and 0.93 for the social entrepreneur variable. The results indicated that social responsibility represents a shared management with the social entrepreneur in companies analyzed, being constructors of sustainable communities in favor of improving the environment wellbeing. It is highlighted as a conclusion that the social entrepreneur must be an integral person from a creative, innovative, tolerant, responsible, visionary approach, and capable to hammer out ideas that help to minimize those effects that affect his voluntary management..
\end{abstract}

Keywords: social responsibility; shared management; social entrepreneur; joint ventures in oil sector; Venezuela.

\section{Introducción}

Los cambios y transformaciones registradas en la última década en los escenarios de la economía venezolana, han conllevado a los sectores productivos de la nación; asimismo al colectivo social al establecimiento de mecanismos de acción participativa voluntaria 
en su entorno, como una alternativa para generar ideas que otorguen respuestas a las realidades acuciantes de la sociedad, de tal manera que se requiere de un trabajo compartido, empresa-comunidad y viceversa, a fin de emprender actividades que agreguen valor y con ello propender la calidad y bienestar de la sociedad con el objeto de garantizar un desarrollo humano sostenible.

En este sentido, las organizaciones en su gestión deben establecer estrategias que guían las acciones tanto administrativas como sociales, no solo con apoyo de su gente sino mediante la participación de las personas de las comunidades aledañas a su ubicación económica, como un compromiso por crear una actuación compartida en las actividades de responsabilidad social, estableciendo una sinergia en el cumplimiento de la dimensión interna y externa, siendo llevadas a cabo a través de modalidades entre ellas: patrocinios, donaciones, becas, uso de instalaciones, jornadas entre otras, estando desarrolladas según la necesidad de las comunidades, representando un comportamiento corresponsable entre los grupos de interés que actúan a favor de consolidar una sociedad más humana y sostenible.

Por consiguiente, al hablarse de la gestión social de las organizaciones que engranan el sistema productivo de un país, este debe fomentarse desde la visión voluntaria que involucre a los grupos de interés, donde las actividades en su administración puedan aportar a resolver los problemas que menoscaben la calidad de vida de las personas asentadas en las comunidades. En virtud de ello, aun cuando la actividad social, sea atribuida a un tema de actualidad este, ha sido una función inserta en su responsabilidad misional, donde no solo es generar bienes y servicios para cumplir los indicadores económicos que sustentan su estabilidad en el mercado, sino también mantener su imagen social como ciudadano corporativo.

En este particular, las organizaciones del campo energético venezolano en este caso las mixtas petroleras, han reestructurado su función económica como primer enlace que suma valor a los indicadores financieros de la nación para destinar el alcance de indicadores sociales, que ayuden al favorecer el bienestar de una sociedad sostenible. Es de esta manera, como la responsabilidad social representa un producto de la ética empresarial, el cual se enlaza en la planificación estratégica organizacional, para afrontar la gestión social compartida, estando llamadas a fomentar gestores de participación integral de su gente con la comunidad, como ente con sentido social responsable.

Cabe señalar, que la responsabilidad social se promueve con la participación e integración voluntaria de los grupos de inte- 
rés, estando apoyada de emprendedores que trabajan por el bien común y un sentido de trabajo en equipo que propicia la formulación y ejecución de ideas hacia la calidad de vida del ser humano. De allí el interés de transformar la práctica social dependiente de entes gubernamentales por la incorporación de una cultura emprendedora en las personas de las comunidades en actividades creativas e innovadoras destinadas a la equidad del colectivo, partiendo de las cualidades que coadyuven mitigar la incertidumbre y desigualdad en la sociedad. De esta manera, el interés en la gestión compartida de las organizaciones petroleras mixtas por el desarrollo de estrategias que contribuyan al bienestar social para una convivencia humana sana.

A razón de lo expuesto, se acota que el emprendedor social representa una persona con característica que lo hacen un ente distintivo, mostrando las competencias, con ideas que lo caracterizan como transformador de los problemas o necesidades del entorno donde interviene, y promoviendo el compromiso responsable en la participación de los colaboradores comunitarios, siendo motores forjadores de una la calidad de vida mejor, de allí el objetivo de los investigadores de analizar la responsabilidad social desde la gestión compartida con el emprendedor social en las empresas mixtas Petroleras Venezolanas.

\section{Responsabilidad social: un enfoque conceptual}

Para Paz, Sánchez y Magdaniel (2015) la responsabilidad social cumple un rol gestor y ejecutor del bienestar en el colectivo social, es decir generador de prosperidad, en los cuales no sólo son protagonistas de su desarrollo sino entes impulsores de calidad de vida del entorno, el cual lo lleva a cabo de manera voluntaria para minimizar efectos que deterioren un ambiente sano.

Según lo expuesto, la responsabilidad social se concibe como las repuestas voluntaria de las organizaciones a las necesidades o problemas surgidos de la sociedad, donde se ofertan los bines y servicios determinados sectores, además debe fomentar el compromiso integral de los grupos de interés a favor de garantizar el bienestar hacia la calidad de Vida. Paz, et al (2015), la responsabilidad social, se concibe como el compromiso voluntario de todos los grupos de interés implementando gestiones que contribuyan con el mejoramiento de las organizaciones en acciones tanto interno como externo, dando lugar a prácticas empresariales transparentes beneficiosas para su talento humano, la sociedad y medio ambiente en el cual están asentadas.

Según Paz, et al (2013), la responsabilidad social constituye una fuente distintiva 
para el alcance de ventajas competitivas y la construcción de un balance ético organizacional, en tal caso las empresas como células de producción social deben comportarse como buenos ciudadanos corporativos, enmarcados en principios éticos, morales, disciplina, igualdad, respeto social y ambiental. En otras palabras, no solo se deben preocupar por el desarrollo organizacional como resultado de su tejido económico sino, ante la formación de la gente hacia una cultura social compartida, el cual incorpore en sus acciones los grupos de interés .

\section{Modalidades de la responsabilidad social}

El sector productivo venezolano han despertado el interés por promover la práctica voluntaria de actividades de responsabilidad social inclusive en la gestión de las empresas del Estado, con iniciativas, que promueven un comportamiento ético, humano, social, y ambiental como parte de su aporte al desarrollo sostenible hacia una sociedad sana. Según Méndez (2003) la sociedad no es un actor especifico objeto de la responsabilidad social, sino una categoría que puede incorporar a distintos actores o grupos, como asociaciones de vecinos, escuelas, centros asistenciales, organizaciones no gubernamentales, iglesias, asociaciones comunitarias, grupos de opinión, asociaciones de productos, universidades, este otros. Para Paz, Sánchez y Magdaniel (2015), las modalidades de la responsabilidad social se relacionan con el tipo de acción social a considerar las empresas, al momento de organizar el desarrollo y ejecución de las actividades en las comunidades, apoyado en el diagnóstico de las necesidades como también en la participación de los grupos de interés.

- Patrocinio: según García (1993, citado en Pelekais y Aguirre 2008), es un mecanismo de cooperación e inversión social representando aportes o fondos otorgados por las empresas condicionados a la obtención de beneficios específicos. Para Capriotti (2007), el patrocinio es el esfuerzo de una organización en asumir la responsabilidad social ante la sociedad.

- Donaciones: Méndez (2003), este puede ser tanto en efectivo como productos o servicios, las primeras son dádivas, regalos y liberalidades; en materia de inversión social, donde se traduce en la entrega de dinero o recursos económicos a organizaciones y a personas como acto altruista de entrega sin esperar nada a cambio; por consiguiente suelen otorgar donaciones a organizaciones no gubernamentales, de manera puntual que no implique dependencia permanente; mientras, las segundas, atinente 
a productos o servicios comprende un instrumento que trata de contribuciones no estructuradas mediante programas o estrategias permanentes. Según Guédez (2006) donaciones se refieren a los aportes de dinero o especies que responden a realidades puntuales y obedecen a un carácter reactivo.

- Premios y becas: Méndez (2003), los premios son galardones o reconocimientos a creaciones, logros educativos, científicos, culturales, deportivos y de trabajo, mientras las becas se asocian a pensiones temporales asignadas a individuos para que realicen estudios; por lo tanto se trata de una forma de contribuir con las personas; buscando lograr la igualdad de oportunidades para el estudio de personas con limitaciones económicas o estímulos a las excelencia académica.

- Voluntariado: Méndez (2003) es la disposición espontanea de una persona para ejecutar un trabajo o servicio sin estar obligado a ello, es un instrumento de cooperación social, de apoyo a terceros. Manifiestan, Gómez y Luis (2007), que la actividad voluntaria es la dimensión proactiva, que busca garantizar la decisión de abordar una iniciativa en el campo de la responsabilidad social de una empresa.
- Usos de Recursos e instalaciones de la empresa: Méndez (2003) es un mecanismo no financiero, ya que asigna o provee de distintos recursos que poseen en la empresa.,

- Mercadeo social o filantrópico: Moreno (2003) señala es una modalidad de apoyo a terceros; utilizando una entidad sin fines de lucros para comercializar determinados productos, asociado a su venta a una donación.

- Cogestión comunal o comunidades locales: Fernández (2005) incluye la integración de las empresas en su entorno local, esta relación de compromiso con la comunidad local se puede evidenciar de formas diversas.

- Relaciones con grupos de interés: Socios Comerciales, Proveedores y Consumidores, para Fernández (2005) el proceso selectivo de socios comerciales, proveedores debe efectuarse con tacto ya que, la relación constituida con ellos puede convertirse en una importante fuente generadora de ventajas en coste y calidad. Asimismo, es recomendable no obviar un factor primordial como lo es la imagen social de la compañía, la cual pudiese resultar perturbada por el accionar de los demás integrantes de la cadena de producción. 
- Derechos humanos: Fernández (2005) afirma que los derechos humanos van más allá de los derechos laborales, los códigos de conducta establecidos a iniciativa de las empresa salvaguardan su imagen, mas no sustituyen a las normas de obligatorio cumplimiento, ya sean legislaciones nacionales, comunitarias o internacionales.

- Problemas ecológicos mundiales: Libro Verde (2004), la disminución del consumo de recursos o de los desechos, así como las emisiones contaminantes puede reducir el impacto sobre el medio ambiente, siendo a su vez beneficiosa para la empresa al reducir sus gastos energéticos, la eliminación de residuos, disminuir los insumos, los gastos de descontaminación.

- Convenios y asociaciones: Méndez (2003) el establecimiento de un sistema de responsabilidad y compromisos entre varios actores mediante la firma de convenio de cooperación en función de la utilidad colectiva; puede darse entre actores diferenciando los roles de financiador y ejecutor, al igual puede dar convenios multiactores que complementan roles de financiamiento, administración y vigilancia.
- Programas sociales y fundaciones corporativas: Para Méndez (2003) los programas son la forma de ejecución directa que asumen ciertas empresas utilizando su estructura funcional, mediante los departamentos de recurso humanos, mercadeo, comunicaciones, relaciones institucionales y asuntos públicos; al igual las fundaciones son un instrumento de inversión social de la empresa, y comprende el apoyo a un conjunto ordenado de acciones, vinculado con la prestación de un servicio, contribuyendo a la solución de problemas sociales, es decir deben responder a las necesidades de la comunidad, generar impacto y garantizar sostenibilidad en un tiempo razonable.

Perea (2011), programa social es un conjunto de proyectos que persiguen los mismos objetivos, que pueden diferenciarse por trabajar con poblaciones diferentes y/o utilizar distintas estrategias de intervención. De esta forma, según las teorías planteadas por los autores los programas sociales son medios empleados por empresas del sector tanto público como privados, para atender las necesidades del entorno social, y con ello contribuir con la calidad de vida, en todo caso promover mediante acciones hacia el bienestar del colectivo social. 


\section{Emprendedor social para el progreso sostenible}

Bruna (2007: 8) "el emprendedor social parte de la premisa el objetivo es la transformación social y medioambiental, construye la organización que permita que esto ocurra, las cuales pueden cerrar con beneficios lucrativos o no lucrativos., suelen abordar los fallos de otros sectores". Paz, et al (2014) los emprendedor social, representa el líder impulsor de la calidad de vida de su gente, mediante innovaciones sociales donde se cubran las necesidades acuciantes de su entorno, mostrando sus capacidades, habilidades, cualidades, destrezas y competencias haciéndolo merecedor de un ser humano distintivo, persistente ante la lucha participativa e incansable por el bienestar de los asuntos sociales de donde convive.

En tal sentido, el papel del emprendedor social, debe ser canalizado por la capacidad de preocuparse en la consecución y diseños de proyecto/organización que forje la calidad de vida del colectivo social mediante esquemas de progreso sostenible, no solo para dar respuestas sobre un determinado sector de la sociedad, sino que los beneficios lleguen de forma objetiva e imparcial a todos para la construcción del desarrollo del bien común. Paz, et al (2012) donde señalan que el protagonismo del emprendedor social al servicio de su gente desde su contexto de ac- ción lo identifica como una persona dispuesta destacando su rol como agente transformador, capaz de involucrar a la comunidad en su gestión para otorgar soluciones sustentables a los problemas o necesidades.

\section{Características del emprendedor social}

De acuerdo con los criterios de Cajina (2014), el emprendedor se caracteriza por conductas que originan buena capacidad organizativa, permitiendo asumir nuevos retos y proyectos e ir más allá de donde ha llegado. Para González (2007), el emprendedor debe mostrar cualidades que los hacer diferentes desde sus características personales, el cual debe poseer iniciativa, capacidad de decisión, acepta el riesgo moderados, orienta hacia la oportunidad, estabilidad emocional, autocontrol , orientación hacia metas específicas, locus de control interno, tolerante, recepciones de las relaciones sociales, sentido de urgencia, honestidad, confianza, perseverancia, responsabilidad personal, individualista y optimista.

$\mathrm{Al}$ igual plantea el autor que entre la cualidades, se hace mención a las físicas, como la energía y trabajo duro, mientras las características intelectuales se atribuye a la versatilidad, creatividad, búsqueda de información, planificador, visión comprensiva, capacidad para de analizar el ambiente, capacidad de solucionar problemas entre otras 
Vainrub (2009:26) "las características de un emprendedor social son iniciativa social, soñadora, atrevida, curiosa, decidido, testaruda, conocedor de sus limitaciones, orientado a tener resultados, capacitado para el cambio, generoso y otros. Son pocos o relativamente pocos, además debe ser un líder". Asimismo, expresan los investigadores que el emprendedor social puede caracterizarse como una persona que fecunda sus acciones agregando valor hacia la transformación de la sociedad, mediante un compromiso compartido con su gente, sustentando sus acciones en valores y principios para solventar problemas que deterioran el bienestar del colectivo, de allí su capacidad creativa, innovadora, tolerante, responsable, visionario y planificador.

- Creativo: para Logan y Logan (1989 citado de Ramos 2006) la creatividad es el potencial del conocimiento y de su desarrollo adecuado, el cual ayuda a percibir el mundo y su propia vida desde un contexto diferente, al desarrollar sus capacidades afectivas, cognoscitivas y perceptivas en las dimensiones desarrollada por ambos hemisferios cerebrales. Por consiguiente, ser creativo implica ser capaz de tener valor y fuerzas para asumir cambios y retos. Por otra parte, Soto y Cárdenas (2007) infieren que los emprendedores sociales son personas creativas para eliminar los obstáculos que se les plantean y capaz de definir sus metas.

- Innovador: Dees, et al (2004) el emprendedor social es un reformador social con visión empresarial que manifiesta una conducta, orienta al desarrollo y surgiendo el espíritu emprendedor interno; genera ideas innovadoras. Ferreiro y Camino (2014) los emprendedores innovadores viven en continua transformación, tanto de sus sistemas productivos como de su organización interna y relación con las demás.

- Tolerante: según Aquiló (2006) es el respeto y consideración hacia la diferencia, como una disposición en los demás una manera de ser y de obrar distinta a la propia, o una actitud de aceptación del legítimo pluralismo.

- Responsable: Soto y Cárdenas (2007) refieren a que la primera responsabilidad del emprendedor es ser una persona ética, desarrollando sus tareas, y cuidando su calidad moral, mientras la segunda responsabilidad es conducir hombres para la acción, para cambiar la realidad, consiguiendo resultados. Para Ramos (2006), la responsabilidad es la capacidad de percibir la obligación. 
- Visionario: para Soto y Cárdenas (2007) el emprendedor social debe poseer una visión compartida, una fuente de creencias en lo que hace, principios y valores sólidos estando éticamente formado. Por otra parte, Austin, Gutiérrez, Ogliastri y Reficco (2006), los emprendedores sociales se caracterizan por ser visionarios, es oportunista más que apegados a un plan o estrategia predeterminada, este se enfoca a construir alianzas y redes de contacto, tendientes a comunicar su visión en términos morales, guiados por el deseo de justicia social más que por el dinero

- Planificador: Munch (2007) el planificar es importante para el adecuado funcionamiento de cualquier grupo social, a través de esta se minimizan riesgos, permitiendo aprovechar los esfuerzos y recursos, reduciendo la incertidumbre, por consiguiente planear, es la busca del rumbo a seguir para operar. Shapero (2007 citado por Ferreiro y Rios 2014), conceptualizan al emprendedor como una persona planificada que toma la iniciativa de organizar unos medios determinados, de modo concreto y para obtener un fin particular.

Desde las perspectivas de Munch (2007) el emprendedor social debe poseer características que les permite mantenerse, entre ellas: valentía, visión, perseverancia, autoconfian- za, orientación hacia a los resultados, liderazgo y creatividad. También señala, el autor que este debe contar con características de personalidad, autocontrol, seguridad de su mismo, iniciativa, sentido común, optimismo, sinceridad, justicia, lealtad, espíritu al logro, visión sencillez y humildad. No obstante, vale acotar que el emprendedor social cree en sus competencias, estando dispuesto a través de su actuación apoyar y pedir ayuda a los grupos de interés de las comunidades para estudiar las oportunidades, colocando de manifiesto sus cualidades para la concepción de ideas u opciones pudiendo resolver los problemas críticos en su entorno.

\section{Metodología}

El presente estudio referente a responsabilidad social y emprendedor social en empresas mixtas petroleras, se cataloga con el paradigma científico, empírico analítico, también conocido como positivista, pragmatista. Señala Guanipa (2010), que el enfoque positivista permite la observación de los hechos sociales con una visión objetiva de la labor científica determinada por una metodología basada en los números, derivada de la recolección de datos mediante la aplicación de cuestionarios tanto válidos como confiables.

En lo referente al tipo de investigación, se asumió como descriptiva, interpretando la caracterización de las variables objeto de in- 
vestigación, mediante la aplicación de técnicas, métodos e instrumentos científicos, conforme con la naturaleza investigativa y los objetivos planteados. Hernández, Fernández y Baptista (2014), la investigación descriptiva busca especificar propiedades, características y rasgos importantes de cualquier fenómeno analizado, describiendo tendencias de un grupo o población, partiendo de perfiles de personas, grupos, comunidades, procesos $\mathrm{u}$ objetos o cualquier otro fenómeno sometido a análisis. Para Méndez (2006), suscribe en aspectos característicos, que identifican diferentes elementos, componentes y su interrelación, teniendo como propósito la delimitación de hechos involucrados en el estudio.

Con respecto a la orientación del objetivo de la investigación, se sustenta de los criterios de una investigación analítica, indagando acerca de los hechos objeto de estudio, según la interpretación del comportamiento de las variables responsabilidad social y emprendedor social en las empresas mixtas petroleras. Para Hurtado (2008) la investigación analítica reinterpreta lo analizado en función de algunos criterios dependientes de los objetivos en estudio. No obstante, considerando la representación del problema observado, en el lugar de investigación, se explica como de campo, donde la información se extrajo en escenarios reales, en este caso las empresas mixtas petroleras. Arias (2006) la investigación de campo recolecta datos direc- tamente en la realidad donde se generan los hechos, sin manipular o controlar variable alguna. Asimismo Tamayo y Tamayo (2010), un diseño de campo, los datos se recogen directamente de la realidad, por lo cual se le denomina primarios.

De acuerdo con el contexto operativo del estudio, y evaluando los objetivos de la investigación se explica desde una estrategia no experimental, donde no se recurrió al análisis y administración de las variables según la manipulación de los datos por los investigadores para estudiar, siendo estas responsabilidad social y emprendedor social en empresas mixtas petroleras, sino la interpretación en el campo de estudio estuvo sujeta a la información recopilada de fuentes primarias y secundarias, para verificar el alcance de los objetivos expuestos. Según el diseño de investigación no experimental, se enuncia en la categoría transversal descriptiva, donde la obtención de información acerca de las variables estudiadas en un momento determinado se desarrollo a juicio de los investigadores, generándose el análisis y descripción de los elementos resaltantes sobre los datos, siendo posteriormente considerando en la redacción de conclusiones en la investigación.

Para efectos de la selección de la población, el estudio se desarrolló considerando, la totalidad de empresas mixtas petroleras del Occidente de la República Bolivariana 
de Venezuela, en este particular las ubicadas geográficamente en el Estado Zulia, representando un total de quince (15) empresas, las cuales se identifican en el cuadro 1:

Cuadro 1

Distribución poblacional de las Empresas Mixtas del sector Petrolero ubicadas en el Occidente de Venezuela-Estado Zulia

\begin{tabular}{lccc}
\hline $\begin{array}{l}\text { Empresas } \\
\text { Mixtas del }\end{array}$ & $\begin{array}{c}\text { Gerentes } \\
\text { de Recurso } \\
\text { Sector Petrolero }\end{array}$ & $\begin{array}{c}\text { Gerentes de } \\
\text { Desarrollo } \\
\text { Social }\end{array}$ & Total \\
\hline Baripetrol & 1 & 1 & 2 \\
Bielovenezolana & 1 & 1 & 2 \\
Petrocumarebo & 1 & 1 & 2 \\
Petroboscan & 1 & 1 & 2 \\
Petrocabimas & 1 & 1 & 2 \\
Petroperijá & 1 & 1 & 2 \\
Petroquiriquire & 1 & 1 & 2 \\
Petro- & 1 & 1 & 2 \\
independiente & & & \\
Petroregional & 1 & 1 & 2 \\
del Lago & 1 & 1 & 2 \\
Lagopetrol & 1 & 1 & 2 \\
Petrowarao & 1 & 1 & 2 \\
Petrowayuu & 1 & 1 & 2 \\
Petrosiven & 1 & & \\
Petrozamora & & & \\
Petrourdaneta & 1 & & \\
Total 30 & & & \\
Gerentes & & & \\
\hline & & & \\
\hline
\end{tabular}

Fuente: Gerencia de Recursos Humanos Empresas Mixtas petroleras de Occidente
Para Méndez (2006) la población es el conjunto total de elementos de estudio. En este sentido, el estudio de hechos o características distintivas acerca del fenómeno estudio, como es el caso de responsabilidad social y emprendedor social se identificó como referencia al personal gerencial de las empresas mixtas petroleras, adscritas a las gerencias de recursos humanos y desarrollo social, siendo constituida por treinta (30) gerentes. En líneas generales, la población gerencial de las empresas objeto de estudio presenta características finitas y accesibles, razón por la que no se acudió a un cálculo muestral, sino se estudió la totalidad de los sujetos de la población, sin señalar efectos discriminativos o características particulares de exclusión, convirtiéndose en un censo poblacional. Méndez (2006) un censo estudia a todos los elementos involucrados en la población, siendo aconsejable aplicarla cuando es finita; asimismo si se requiere información inmediata del estudio.

Para la técnica, fueron considerados los medios para obtener la información sobre las variables investigadas, se aplicó como técnica la encuesta, Méndez (2006; citado de Tamayo y Tamayo 2001) la encuesta es un instrumento de observación formado por una serie de preguntas formuladas, cuyas respuestas son anotadas por el en- 
cuestador, permitiendo el conocimiento de las motivaciones, actitudes y opiniones de los individuos con relación de su objeto de investigación. Sabino (2008), es la obtención de información de personas acerca de los problemas en estudio para luego, mediante un análisis de tipo cuantitativo, emitir conclusiones que correspondan con los datos recogidos.

Con relación al instrumento, utilizado en la recolección de datos, se aplicó un cuestionario, pertinente a 35 ítems para la variable responsabilidad social y 18 ítems emprendedor social, mediante la emisión de respuestas múltiples de siempre, casi siempre, a veces, casi nunca y nunca para ambas variables. Méndez (2006) un cuestionario está constituido por un conjunto de preguntas, donde el investigador precisa la información que pretende para el trabajo. Coincidiendo Sabino (2008), que es un instrumento concreto de recolección de información que contiene un conjunto de ítems. Cabe destacar, que los instrumentos se validaron por juicios de 10 expertos y aplicando la confiabilidad por la formula de Alfa de Cronbach, dando un resultado de 0,94 para la variable responsabilidad social, mientras el emprendedor social 0,93 , considerándose instrumentos confiables.

\section{Resultados}

Según los argumentos expuestos, se presenta el análisis los resultados, representando la integración cuantitativa de los datos arrojados de la opinión de los encuestados, razón por la cual, a través de la información recopilada, previamente codificada y tabulada, se discuten e interpretan los resultados obtenidos en la investigación, según los objetivos desglosados en dimensiones e indicadores para medir el comportamiento de las variables estudiadas.

Cuadro 2

Baremo de interpretación del promedio de datos

\begin{tabular}{cc}
\hline Rangos & Categorías \\
\hline $1,00 \geq 1,80$ & Muy baja \\
$1,81 \geq 2,60$ & Baja \\
$2,61 \geq 3,40$ & Moderada \\
$3,41 \geq 4,20$ & Alta \\
$4,21 \geq 5,00$ & Muy alta \\
\hline
\end{tabular}

Fuente: Paz, Harris y Franco (2015)

Objetivo: analizar la responsabilidad social como gestión compartida con el emprendedor social en empresas mixtas del sector petrolero venezolano. Dimensión: Modalidades de la responsabilidad social 
Tabla 1

Modalidades de la Responsabilidad Social

\begin{tabular}{|c|c|c|c|c|c|c|c|c|c|c|c|c|c|c|}
\hline \multirow{3}{*}{ Indicadores } & \multirow{3}{*}{$\begin{array}{c}\begin{array}{c}\text { Alternativas } \\
\text { de resp. }\end{array} \\
\text { Ítems }\end{array}$} & \multicolumn{2}{|c|}{$\mathrm{S}$} & \multicolumn{2}{|c|}{$\mathrm{Cs}$} & \multirow{2}{*}{\multicolumn{2}{|c|}{$\frac{\mathrm{Av}}{\text { Gerentes }}$}} & \multicolumn{2}{|c|}{$\mathrm{Cn}$} & \multicolumn{2}{|c|}{$\mathrm{N}$} & \multirow{2}{*}{\multicolumn{3}{|c|}{ Promedios }} \\
\hline & & & & & & & & & & & & & & \\
\hline & & $\mathrm{Fa}$ & Fr\% & $\mathrm{Fa}$ & $\mathrm{Fr} \%$ & $\mathrm{Fa}$ & Fr\% & $\mathrm{Fa}$ & $\mathrm{Fr} \%$ & $\mathrm{Fa}$ & Fr\% & Ítem & Ind. & Dim. \\
\hline \multirow{3}{*}{ Patrocinio } & 1 & 20 & $67 \%$ & 5 & $17 \%$ & 5 & $17 \%$ & 0 & $0 \%$ & 0 & $0 \%$ & 4,50 & \multirow{3}{*}{4,0} & \multirow{22}{*}{3,9} \\
\hline & 2 & 0 & $0 \%$ & 22 & $73 \%$ & 8 & $27 \%$ & 0 & $0 \%$ & 0 & $0 \%$ & 3,73 & & \\
\hline & 3 & 0 & $0 \%$ & 25 & $83 \%$ & 3 & $10 \%$ & 2 & $7 \%$ & 0 & $0 \%$ & 3,77 & & \\
\hline \multirow{3}{*}{ Donaciones } & 4 & 0 & $0 \%$ & 0 & $0 \%$ & 20 & $67 \%$ & 5 & $17 \%$ & 5 & $17 \%$ & 2,50 & \multirow{3}{*}{3,2} & \\
\hline & 5 & 0 & $0 \%$ & 23 & $77 \%$ & 3 & $10 \%$ & 4 & $13 \%$ & 0 & $0 \%$ & 3,63 & & \\
\hline & 6 & 0 & $0 \%$ & 18 & $60 \%$ & 8 & $27 \%$ & 2 & $7 \%$ & 2 & $7 \%$ & 3,40 & & \\
\hline \multirow{3}{*}{$\begin{array}{c}\text { Premio y } \\
\text { Becas }\end{array}$} & 7 & 6 & $20 \%$ & 20 & $67 \%$ & 2 & $7 \%$ & 2 & $7 \%$ & 0 & $0 \%$ & 4,00 & \multirow{3}{*}{3,5} & \\
\hline & 8 & 0 & $0 \%$ & 5 & $17 \%$ & 15 & $50 \%$ & 6 & $20 \%$ & 4 & $13 \%$ & 2,70 & & \\
\hline & 9 & 0 & $0 \%$ & 4 & $13 \%$ & 16 & $53 \%$ & 10 & $33 \%$ & 0 & $0 \%$ & 3,87 & & \\
\hline \multirow{3}{*}{ Voluntariado } & 10 & 2 & $7 \%$ & 22 & $73 \%$ & 5 & $17 \%$ & 0 & $0 \%$ & 1 & $3 \%$ & 3,80 & \multirow{3}{*}{3,9} & \\
\hline & 11 & 0 & $0 \%$ & 27 & $90 \%$ & 3 & $10 \%$ & 0 & $0 \%$ & 0 & $0 \%$ & 3,90 & & \\
\hline & 12 & 0 & $0 \%$ & 26 & $87 \%$ & 4 & $13 \%$ & 0 & $0 \%$ & 0 & $0 \%$ & 3,87 & & \\
\hline \multirow{3}{*}{$\begin{array}{l}\text { Uso de } \\
\text { Instalaciones }\end{array}$} & 13 & 0 & $0 \%$ & 10 & $33 \%$ & 18 & $60 \%$ & 2 & $7 \%$ & 0 & $0 \%$ & 3,27 & \multirow{3}{*}{3,5} & \\
\hline & 14 & 0 & $0 \%$ & 10 & $33 \%$ & 16 & $53 \%$ & 2 & $7 \%$ & 2 & $7 \%$ & 3,13 & & \\
\hline & 15 & 10 & $33 \%$ & 14 & $47 \%$ & 4 & $13 \%$ & 2 & $7 \%$ & 0 & $0 \%$ & 4,07 & & \\
\hline \multirow{3}{*}{$\begin{array}{l}\text { Mercado } \\
\text { Social }\end{array}$} & 16 & 10 & $33 \%$ & 10 & $33 \%$ & 10 & $33 \%$ & 0 & $0 \%$ & 0 & $0 \%$ & 4,00 & \multirow{3}{*}{3,9} & \\
\hline & 17 & 18 & $60 \%$ & 2 & $7 \%$ & 10 & $33 \%$ & 0 & $0 \%$ & 0 & $0 \%$ & 4,27 & & \\
\hline & 18 & 8 & $27 \%$ & 8 & $27 \%$ & 8 & $27 \%$ & 2 & $7 \%$ & 4 & $13 \%$ & 3,47 & & \\
\hline \multirow{4}{*}{$\begin{array}{l}\text { Cogestión } \\
\text { Comunal }\end{array}$} & 19 & 19 & $63 \%$ & 5 & $17 \%$ & 6 & $20 \%$ & 0 & $0 \%$ & 0 & $0 \%$ & 4,43 & \multirow{4}{*}{4,3} & \\
\hline & 20 & 16 & $53 \%$ & 9 & $30 \%$ & 5 & $17 \%$ & 0 & $0 \%$ & 0 & $0 \%$ & 4,37 & & \\
\hline & 21 & 12 & $40 \%$ & 10 & $33 \%$ & 4 & $13 \%$ & 4 & $13 \%$ & 0 & $0 \%$ & 4,00 & & \\
\hline & 22 & 15 & $50 \%$ & 15 & $50 \%$ & 0 & $0 \%$ & 0 & $0 \%$ & 0 & $0 \%$ & 4,50 & & \\
\hline
\end{tabular}


Cont...

\begin{tabular}{|c|c|c|c|c|c|c|c|c|c|c|c|c|c|c|}
\hline \multirow{3}{*}{ Indicadores } & \multirow{3}{*}{$\begin{array}{c}\begin{array}{c}\text { Alternativas } \\
\text { de resp. }\end{array} \\
\text { Ítems } \\
\end{array}$} & \multicolumn{2}{|c|}{$\mathrm{S}$} & \multicolumn{2}{|c|}{ Cs } & \multicolumn{2}{|c|}{$\mathrm{Av}$} & \multicolumn{2}{|c|}{$\mathrm{Cn}$} & \multicolumn{2}{|c|}{$\mathrm{N}$} & \multirow{2}{*}{\multicolumn{3}{|c|}{ Promedios }} \\
\hline & & \multicolumn{10}{|c|}{ Gerentes } & & & \\
\hline & & $\mathrm{Fa}$ & $\mathbf{F r} \%$ & $\mathbf{F a}$ & Fr\% & $\mathbf{F a}$ & Fr\% & $\mathbf{F a}$ & Fr\% & $\mathrm{Fa}$ & Fr\% & Ítem & Ind. & Dim. \\
\hline \multirow{3}{*}{$\begin{array}{c}\text { Relaciones } \\
\text { con Grupos } \\
\text { de Interés }\end{array}$} & 23 & 13 & $43 \%$ & 8 & $27 \%$ & 4 & $13 \%$ & 5 & $17 \%$ & 0 & $0 \%$ & 3,97 & \multirow{3}{*}{3,8} & \\
\hline & 24 & 7 & $23 \%$ & 10 & $33 \%$ & 10 & $33 \%$ & 3 & $10 \%$ & 0 & $0 \%$ & 3,70 & & \\
\hline & 25 & 9 & $30 \%$ & 8 & $27 \%$ & 13 & $43 \%$ & 0 & $0 \%$ & 0 & $0 \%$ & 3,87 & & \\
\hline \multirow{3}{*}{$\begin{array}{l}\text { Derechos } \\
\text { Humanos }\end{array}$} & 26 & 14 & $47 \%$ & 5 & $17 \%$ & 5 & $17 \%$ & 5 & $17 \%$ & 1 & $3 \%$ & 3,87 & \multirow{3}{*}{3,9} & \\
\hline & 27 & 13 & $43 \%$ & 16 & $53 \%$ & 1 & $3 \%$ & 0 & $0 \%$ & 0 & $0 \%$ & 4,40 & & \\
\hline & 28 & 10 & $33 \%$ & 8 & $27 \%$ & 2 & $7 \%$ & 5 & $17 \%$ & 5 & $17 \%$ & 3,43 & & \\
\hline \multirow{3}{*}{$\begin{array}{l}\text { Problemas } \\
\text { Ecológicos } \\
\text { Mundiales }\end{array}$} & 29 & 15 & $50 \%$ & 15 & $50 \%$ & 0 & $0 \%$ & 0 & $0 \%$ & 0 & $0 \%$ & 4,50 & \multirow{4}{*}{4,5} & 3.9 \\
\hline & 30 & 17 & $57 \%$ & 10 & $33 \%$ & 3 & $10 \%$ & 0 & $0 \%$ & 0 & $0 \%$ & 4,47 & & \\
\hline & 31 & 18 & $60 \%$ & 11 & $37 \%$ & 1 & $3 \%$ & 0 & $0 \%$ & 0 & $0 \%$ & 4,57 & & \\
\hline \multirow{2}{*}{$\begin{array}{l}\text { Convenios y } \\
\text { Asociaciones }\end{array}$} & 32 & 15 & $50 \%$ & 15 & $50 \%$ & 0 & $0 \%$ & 0 & $0 \%$ & 0 & $0 \%$ & 4,50 & & \\
\hline & 33 & 10 & $33 \%$ & 19 & $63 \%$ & 1 & $3 \%$ & 0 & $0 \%$ & 0 & $0 \%$ & 4,30 & \multirow{3}{*}{4,1} & \\
\hline $\begin{array}{l}\text { Programa } \\
\text { Sociales y }\end{array}$ & 34 & 9 & $30 \%$ & 10 & $33 \%$ & 11 & $37 \%$ & 0 & $0 \%$ & 0 & $0 \%$ & 3,93 & & \\
\hline $\begin{array}{l}\text { Fundaciones } \\
\text { Corporativas }\end{array}$ & 35 & 10 & $33 \%$ & 11 & $37 \%$ & 8 & $27 \%$ & 1 & $3 \%$ & 0 & $0 \%$ & 4,00 & & \\
\hline
\end{tabular}

Fuente: Paz, Harris y Franco (2015)

Con relación a los datos ilustrados en la tabla 1 se puede observar que los resultados relacionados con las modalidades de la responsabilidad social en empresas mixtas petroleras, se conoció en función al indicador patrocinio, los encuestados expresaron con un $87 \%$ que las empresas apoyan los programas educativos desarrollados en la comunidad; en relación a do- naciones un $77 \%$ destacó la participación de casi siempre las empresas estudiadas apoyan la iniciativa de organismos locales destinados al desarrollo de programas sociales; en referencia a premios y becas un $67 \%$ señalo recibir reconocimientos por los logros alcanzados de su formación educativa. 
Para la medición del indicador voluntariado, un 90\% de los encuestados refirieron que casi siempre cuando no ejercen su función voluntaria en la participación el empleado en actividades sociales son asignados por la gerencia; en cuanto al uso de instalaciones el $60 \%$ se pronunció que a veces los consejos comunales recurren al apoyo de su instalaciones para efectuar actividades de interés social; el mercadeo social se vio medido con un $60 \%$ de los encuestados al expresar que siempre recurren a la publicidad para dar a conocer las actividades sociales de la empresa.

Para los efectos de la cogestión comunal manifestaron los encuestados con un $60 \%$ que evalúan siempre las exigencia de la comunidad de acuerdo con sus necesidades, la relación con los grupos de interés se detectaron aspectos discrepantes, ya que siempre participan con un $43 \%$ según opinión de los encuestados participan con otras empresas en actividades sociales, y a veces también con un $43 \%$ se integran de forma conjunta ante la comunidad. La medición del indicador derecho humano un $53 \%$ menciono que se difunde a veces entre los empleados lo contemplado en la ley orgánica del trabajo, trabajador y trabajadora.

En cuanto a los problemas ecológicos un $60 \%$ mostro que promueven siempre campañas ambientalistas en la comunidad; del mismo modo un $63 \%$ plantearon apoyarse en convenios con sector privados para desarrollar actividades socio-ambientales; asimismo se promueve casi siempre con un 37 \% la ejecución de actividades sociales en su comunidad aquellos elaborados por los empleados para atender las necesidades de la comunidad. A modo general, se percibe que el promedio más alto entre los indicadores se ubicó en los problemas ecológicos mundiales con un 4,5, siendo muy alta responsabilidad, mientras el promedio de la dimensión es atribuyo a un 3,9 siendo según la categoría del baremo de interpretación de medias como alta la responsabilidad de las empresas mixtas en actividades de responsabilidad social.

Los datos obtenidos de la opinión emitida por los encuestados, referente a las características del emprendedor social en empresas mixtas petroleras, se conoció que el indicador creativo se manifestó en un $53 \%$ casi siempre dialoga con las empresas para brindar iniciativas que den respuesta a la comunidad, el indicador innovador un $47 \%$ expreso siempre y casi siempre sus aportes son escuchados en la comunidad para canalizar los problemas frente a los organismos encargados de solventar sus necesidades. Con respecto, al indicador tolerante, el 93\% afirma siempre de acuerdo, muestran descontento en la comunidad cuando no son solventados los problemas 
Tabla 2

Características del emprendedor social.

\begin{tabular}{|c|c|c|c|c|c|c|c|c|c|c|c|c|c|c|}
\hline \multirow{3}{*}{ Indicadores } & \multirow{3}{*}{$\begin{array}{c}\begin{array}{c}\text { Alternativa } \\
\text { de Resp. }\end{array} \\
\text { Ítems }\end{array}$} & \multirow{2}{*}{\multicolumn{2}{|c|}{$\mathrm{S}$}} & \multicolumn{2}{|c|}{ CS } & \multicolumn{2}{|c|}{$\mathrm{AV}$} & \multicolumn{2}{|c|}{$\mathrm{CN}$} & \multicolumn{2}{|c|}{$\mathrm{N}$} & \multirow{2}{*}{\multicolumn{3}{|c|}{ Promedios }} \\
\hline & & & & & & Ger & entes & & & & & & & \\
\hline & & $\mathrm{Fa}$ & Fr\% & $\mathbf{F a}$ & Fr\% & $\mathrm{Fa}$ & Fr\% & $\mathbf{F a}$ & Fr\% & $\mathbf{F a}$ & Fr\% & Ítem & Ind. & Dim. \\
\hline \multirow{3}{*}{ Creativo } & 1 & 10 & $33 \%$ & 12 & $40 \%$ & 8 & $27 \%$ & 0 & $0 \%$ & 0 & $0 \%$ & 4,07 & \multirow{3}{*}{4,1} & \multirow{18}{*}{4,3} \\
\hline & 2 & 9 & $30 \%$ & 13 & $43 \%$ & 6 & $20 \%$ & 2 & $7 \%$ & 0 & $0 \%$ & 3,97 & & \\
\hline & 3 & 6 & $20 \%$ & 16 & $53 \%$ & 6 & $20 \%$ & 0 & $0 \%$ & 2 & $7 \%$ & 3,80 & & \\
\hline \multirow{3}{*}{ Innovador } & 4 & 8 & $27 \%$ & 12 & $40 \%$ & 10 & $33 \%$ & 0 & $0 \%$ & 0 & $0 \%$ & 3,93 & \multirow{3}{*}{3,9} & \\
\hline & 5 & 14 & $47 \%$ & 14 & $47 \%$ & 2 & $7 \%$ & 0 & $0 \%$ & 0 & $0 \%$ & 4,40 & & \\
\hline & 6 & 15 & $50 \%$ & 10 & $33 \%$ & 5 & $17 \%$ & 0 & $0 \%$ & 0 & $0 \%$ & 4,33 & & \\
\hline \multirow{3}{*}{ Tolerante } & 7 & 25 & $83 \%$ & 5 & $17 \%$ & 0 & $0 \%$ & 0 & $0 \%$ & 0 & $0 \%$ & 4,83 & & \\
\hline & 8 & 22 & $73 \%$ & 8 & $27 \%$ & 0 & $0 \%$ & 0 & $0 \%$ & 0 & $0 \%$ & 4,73 & 4,8 & \\
\hline & 9 & 28 & $93 \%$ & 0 & $0 \%$ & 2 & $7 \%$ & 0 & $0 \%$ & 0 & $0 \%$ & 4,87 & & \\
\hline \multirow{3}{*}{ Responsable } & 10 & 21 & $70 \%$ & 7 & $23 \%$ & 2 & $7 \%$ & 0 & $0 \%$ & 0 & $0 \%$ & 4,63 & \multirow{3}{*}{4,6} & \\
\hline & 11 & 13 & $43 \%$ & 10 & $33 \%$ & 5 & $17 \%$ & 2 & $7 \%$ & 0 & $0 \%$ & 4,13 & & \\
\hline & 12 & 15 & $50 \%$ & 6 & $20 \%$ & 7 & $23 \%$ & 2 & $7 \%$ & 0 & $0 \%$ & 4,13 & & \\
\hline \multirow{3}{*}{ Visionario } & 13 & 24 & $80 \%$ & 5 & $17 \%$ & 0 & $0 \%$ & 0 & $0 \%$ & 1 & $3 \%$ & 4,70 & \multirow{3}{*}{4,7} & \\
\hline & 14 & 17 & $57 \%$ & 10 & $33 \%$ & 3 & $10 \%$ & 0 & $0 \%$ & 0 & $0 \%$ & 4,47 & & \\
\hline & 15 & 15 & $50 \%$ & 15 & $50 \%$ & 0 & $0 \%$ & 0 & $0 \%$ & 0 & $0 \%$ & 4,50 & & \\
\hline \multirow{3}{*}{$\begin{array}{l}\text { Capaz de } \\
\text { planificar }\end{array}$} & 16 & 0 & $0 \%$ & 18 & $60 \%$ & 10 & $33 \%$ & 0 & $0 \%$ & 2 & $7 \%$ & 3,47 & \multirow{3}{*}{3,5} & \\
\hline & 17 & 15 & $50 \%$ & 15 & $50 \%$ & 0 & $0 \%$ & 0 & $0 \%$ & 0 & $0 \%$ & 4,50 & & \\
\hline & 18 & 2 & $7 \%$ & 22 & $73 \%$ & 6 & $20 \%$ & 0 & $0 \%$ & 0 & $0 \%$ & 3,87 & & \\
\hline
\end{tabular}

Fuente: Paz, Harris y Franco (2015) 
Lo relacionado con el indicador responsable se vio medido por un $70 \%$ de los encuestados al destacar dialogar con la comunidad cuando percibe que no se están haciendo las cosas como se planificaron; con respecto al indicador visionario un $80 \%$ respondió que recurren al apoyo de empresas privadas para prever el incumplimiento de actividades de interés social planificadas por otros organismos; el indicador planificador un $60 \%$ indico que casi siempre utiliza la planificación de tareas entre la gente de la comunidad para dar respuesta a sus necesidades. A modo general, la dimensión tuvo como resultado promedio más alto al indicador tolerante con un 4,8 siendo caracterizada por la categoría altamente desarrollado el emprendedor social, mientras la media arrojada como resultado de la dimensión fue de 4,3 siendo ubicada según el baremo de interpretación de resultados promedios en la categoría altamente desarrollada coincidiendo con el valor suscrito para el indicador.

\section{Conclusiones}

El analizar la responsabilidad social como gestión compartida con el emprendedor social en las empresas mixtas petroleras de Venezuela, permitió describir la situación objeto de estudio, con referencia a las modalidades del desarrollo de programas educa- tivos en la comunidad donde está asentada su actividad económica, asumiendo al igual la participación de actividades culturales y deportivas en pro de aportar a la mejora de la calidad de vida del socio colectivo, mediante donaciones, conjugando además su actuación responsable con el establecimiento de alianzas con organismos públicos locales y otra empresas privadas del sector adyacente a su entorno. Por consiguiente, se conoció el otorgamiento de reconocimientos a su gente por resultados alcanzados de la formación profesional; asimismo se otorgan becas a sus familiares directos, y en casos particulares mediante convocatorias o sugerencias del consejo comunal en la comunidad.

Por otra parte, vale destacar que se promueve en las empresas objeto de estudio el voluntariado social en los colaboradores de su fuerza laboral, para desarrollar aportes significativos hacia las comunidades; del mismo modo se orientan a los consejos comunales utilizando las instalaciones de ellas, sin que esto interrumpa su actividades tanto administrativas como operativas, esto con el objeto de establecer un dialogo que sirva de fuente informativa para conocer sus necesidades acuciantes, integrando a los grupos de interés, del mismo modo apoyarse siendo portavoces de los problemas ecológicos, convenios o alianzas que se establecen, destacando la formulación 
de programas sociales que les coadyuvan su interés por elevar la calidad de vida.

Con relación a las características del emprendedor social en empresas mixtas petroleras, los resultaron indicaron que el emprendedor social debe es una persona integral desde un enfoque creativo, innovador, tolerante, responsable, visionario, capaz de forjar ideas que faciliten no solo la planificación de ideas a desarrollar hacia la calidad de vida y bienestar del colectivo social en sus comunidades, sino ser agente transformador del cambio para minimizar efectos que incidan sobre su gestión voluntaria.

Por otra parte, como parte de las principales contribuciones desde un orden científico-práctico se destacan producto del trabajo investigativo, lo siguiente:

- Mantener el apoyo de los patrocinios en el campo social, educativo, deportivos y otros, en las comunidades con la participación integral de los grupos de interés, a objeto de instaurar estrategias de integración empresa - sociedad y viceversa, donde mediante talleres de sensibilidad social se expongan las necesidades desde el dialogo participativo para sugerir acciones que faciliten la incorporación de iniciativas creativas e innovadoras acorde a la situación problema.
- Promover la integración de la comunidad en programas sociales desarrollados por entes públicos y privados, para generar planes de acción cónsonos con sus realidades, y permitir captar e intercambiar ideas sobre sus necesidades, para el fortalecimiento hacia el bienestar y calidad de vida de las comunidades, con la participación de los colaboradores tanto internos como externos a la organización, para orientar a la comunidad en la planificación de actividades de trabajo compartido y generar compromiso con la comunidad, al igual que facilitar el seguimiento al desarrollo de la inversión en los programas sociales, previa formación de la participación de emprendedores que sean el líder responsable de su entorno.

- Difundir en el entorno la importancia del emprendedor social en la comunidad, mediante acciones de orientación que facilita el desarrollo y fortalecimiento de sus capacidades creativas, tolerante, responsable, visionario, innovador, a razón de gestar una cultura de responsabilidad social congruente para detectar las necesidades del colectivo social, y con ello garantizar la calidad de vida de su gente. 
- La integración para conservar la capacidad emprendedora de líderes comunitarios, que otorguen respuestas reales a las comunidades, bajo premisas de desempeño con los grupos de interés que coadyuven la gestión compartida. De igual modo, promover la captación de ideas en las comunidades como alternativa de respuestas a sus necesidades o problemas, mediante convocatorias que faciliten explorar nuevas oportunidades para la transformación de su entorno, dando a conocer las respuestas a través de diálogos participativos en la construcción de la convivencia justa.

\section{Referencias}

Aquiló, A. (2005). La tolerancia. España: Editorial Palabras.

Arias, F. (2006). El Proyecto de Investigación. Venezuela: Editorial Episteme.

Austin, J., Gutiérrez, R., Ogliastri, E. y Reficco, E. (2006). Gestión efectiva de emprendimientos sociales. Lecciones extraidas de empresas y organizaciones de la sociedad civil en Iberoamérica. Un Proyecto de Investigación Colectiva de la Social Enterprise Knowledge Network. Banco Interamericano de Desarrollo David Rockefeller Center forLatin American Studies Harvard University Washington, D.C., 2006.
Bruna, F. (2007). Creación de empresas de económica social. España: Editorial Ideas propias.

Cajina, G. (2014). Coaching para emprender. Cambia tu rumbo, despide a tu jefe. México, D. F.: Ediciones Díaz de Santos.

Capriotti, P. (2007). El patrocinio como expresión de la responsabilidad social de una organización. Revista razón y palabra. 56 .

Dees, J., Anderson, B. y WEI Skillern, J. (2004). Escala de impacto social: estrategias para la difusión de la innovación social. Stanford Social Innovation Review 1 (4), pp. 24-32.

Ferreiro, F. y Camino, M. (2014). La contribución del mundo empresarial en los modelos de crecimiento económico. $4^{\mathrm{a}}$ Conferencia Ibérica de Emprendimiento. Pontevedra, España.

Ferreiro, F. y Rios, D. (2014). El concepto de emprendedor y la empresa familiar. $4^{a}$ Conferencia Ibérica de Emprendimiento. Pontevedra, España 23 al 26 de octubre de 2014.

Fernández, R. (2005). Administración de la Responsabilidad Social Corporativa. España: Editorial internacional Thompson Ediciones. 
Gómez, H. y Luis-Bassa, C. (2007). Iniciativa social como estrategia competitiva. Caracas: Ediciones Instituto de Estudios de Administración (IESA).

González, D. (2007). Plan de negocios para emprendedores al éxito. México, D. F.: Editorial McGrawHill.

Guanipa, M. (2010). Reflexiones básicas sobre investigación. Maracaibo: Editorial de la Universidad Rafael Belloso Chacín.

Hernández, R., Fernández, C. y Baptista, M. (2014). Metodología de la Investigación. México, D. F.: Editorial Mc. Graw Hill Interamericana Editores.

Hurtado, J. (2008). Metodología de la Investigación. Venezuela: Fundación Sypal. Editorial Magisterio

Méndez, C. (2006). Metodología Diseño y Desarrollo del Proceso de Investigación. Colombia: Editorial Limusa.

Méndez, Ch. (2003). Responsabilidad Social de Empresarios y Empresas en Venezuela durante el siglo XXI. Venezuela: Editorial Strategos Consultores.

Munch, L. (2007). Administración. Escuelas, proceso administrativo, áreas funciomnales y desarrollo emprendedor. México, D.F.: Editorial Pearson Educación.
Paz, A., Sánchez, J. y Magdaniel, Y. (2015). Responsabilidad social: un compromiso de todos. Colombia: Editorial Universidad de La Guajira.

Paz, A., Paz, E. y Franco, F. (2012). El emprendedor social como eje transformador de las comunidades venezolanas. IV Jornadas Científicas Nacionales Dr. José Gregorio Hernández. "Valores en la Universidad: Elemento transformacional para una sociedad ética y equitativa”. Maracaibo/Venezuela.

Paz, A., Paz, J. y Franco, F. (2013). Responsabilidad social: una mirada sustentable hacia al ambiente en empresas mixtas petroleras. V Jornadas Científicas Nacionales Dr. José Gregorio Hernández "Encuentro de Saberes Universitarios”. Maracaibo/Venezuela.

Paz, A., Sánchez, J. y Pedraza, C. (2014). Emprendedor social: constructor de integración de la responsabilidad social universitaria con la comunidad. I Jornada Binacional de Investigación Maracaibo. Venezuela. Universidad Rafael Belloso Chacin.

Pelekais, C. y Aguirre, R. (2008). Hacia Una Cultura de Responsabilidad Social. México, D.F.: Ediciones Pearson. 
Perea, L. (2011). La responsabilidad social de la empresa en América Latina. Capítulo 8: Las instituciones del mercado financiero. New York. EEUU. Publicación del Banco Interamericano de Desarrollo, Mayo 2011

Ramos, M. (2006). Valores y autoestima. Conociéndose a sí mismo en un mundo de otros. Venezuela: Editorial San Pablo.
Sabino, C. (2008). El proceso de investigación. Caracas: Editorial Panapo

Soto, E. y Cárdenas, J. (2007). Ética en las Organizaciones. México, D. F.: Editorial McGraw-Hill Interamericana.

Tamayo y Tamayo, M. (2010). El Proceso de la Investigación Científica. México D. F.: Editorial Limusa.

Vainrub, R. (2009). Una guía de emprendedores sociales. México, D.F.: Pearson. 